\title{
The Impact of Influenza in COVID-19 Pandemic
}

\section{Masoud Mardani (iD ${ }^{1, *}$}

${ }^{1}$ Infectious Diseases and Tropical Medicine Research Center, Shahid Beheshti University of Medical Sciences, Tehran, Iran

"Corresponding author: Infectious Diseases and Tropical Medicine Research Center, Shahid Beheshti University of Medical Sciences, Tehran, Iran. Tel: +98-9121132678, Email: drmasoudmardani@yahoo.com

Received 2021 March 07; Accepted 2021 March 07.

Keywords: Influenza, COVID-19

There are several questions concerning the association between flu season and the COVID-19 pandemic. For instance, does the influenza coinfection affects the clinical course of COVID-19? There are also uncertainties regarding the impact of influenza vaccination on preventing COVID19 infection or whether COVID-19 preventive measures are associated with the reduced burden of the coming flu season (1). Some points are derived from studies performed in China, where influenza was extensively transmitting when the first cases of novel coronavirus were identified, and in the southern hemisphere that still is in the midst of flu season (1).

Each year the influenza epidemic imposes significant effects on health systems all around the world, which claims about 12000 to 61000 lives each year since 2010 just in the US (2). It has been argued that the total number of deaths and infected cases is a criterion of the degree of genetic drift or shift in the dominant strain of the influenza virus. Besides, somehow, it reflects the efficacy of vaccination. Since the onset of the COVID-19 pandemic, health care providers are faced with an increased burden of respiratory problems, somehow due to its expansion in an immunologically naive population. Currently, one of the most important concerns of health authorities is the looming threat of concurrent influenza and COVID-19 epidemics (3).

Severe acute respiratory syndrome coronavirus 2 (SARS-CoV-2) and influenza have major pathogenic differences. Nevertheless, there are key areas of overlap (3-6). For instance, both are mainly transmitted airborne. Hence, using non-pharmacological interventions (NPIs), like face covering, closure of schools and other crowded areas, and restrictions on unnecessary movements, can decline the expansion rate of both infections to varying degrees. Several studies reported decreased incidence rate for influenza in 2020 (January through May) following the implementation of NPIs $(5,6)$. A similar trend is found in the US. Caution should be taken when interpreting such findings. As early as the pandemic, the testing equipment was not available everywhere (2).

The assumption that the declined incidence of influenza incidence will continue during the coming influenza season reflects continuing adherence to NPIs. Long-term use of protective equipment and imposing lockdowns when the incidence of the disease increases have declined the rates of infection for both influenza and COVID19. However, simultaneous with relaxing restrictions, the incidence of both influenza and COVID-19 will increase (2).

Apart from NPIs, controlling seasonal influenza through vaccination is important for declining the viral reservoir. Despite the widespread accessibility of different vaccines for influenza, national coverage of influenza vaccination is consistently lower than $50 \%$ in adult populations. Combining national awareness campaigns and community-based vaccination programs focused on highrisk populations and historically deprived populations is of crucial importance for enhancing the population's health (2).

At least two issues are clear: (A) Testing infrastructures should be expanded more widely to differentiate these two viral infections. It worth noting that although these infections have similar presentations, at least at first, but require various therapeutic options. Furthermore, a severe influenza season-due to more virulent strains or insufficient vaccination- added to the COVID-19 pandemic, with no sign of abating, could overwhelm already taxed emergency wards and intensive care units (1).

Each of these viruses requires a different treatment strategy. The main therapeutic option for influenza is neuraminidase inhibitors or a cap-dependent endonuclease inhibitor, which do not carry antiviral activities against COVID-19. On the other hand, one of the most common treatments for COVID-19 patients is remdesivir, but due to its parental administration, it is restricted to inpatients. It is also necessary to approve a COVID-19 diagnosis to facilitate early engagement in trials, particularly for those 
with remdesivir contraindications. Several other therapeutic options for COVID-19 are under trial, like oral antivirals that may have brought significant changes for managing outpatient cases (2). Afterward, each virus causes a different clinical course. Those infected with influenza often develop the most severe signs during the first days of infection, while those infected with COVID-19 mostly have a longer period of presentation with a peak at the second or third week of infection. Differentiating infections caused by each of these viruses allows health care providers to better decisions about treatment strategies, which is also useful for the identification of the following complications (2). Furthermore, the precise diagnosis is of crucial importance for disease management, such as standard guidelines for quarantine, opening schools and backing to work, and identifying COVID-19 patients and contact tracing (2). To sum up, in Iran, due to the expected outbreak of influenza in the last months of summer, as well as increased hospitalization and higher occupancy of ECU beds due to the recent pandemic, particular attention should be paid to the co-occurrence of influenza and COVID-19. Hence, health authorities should pay more attention to this issue, in addition to controlling the epidemic, which can decline both morbidity and mortality.

\section{Footnotes}

Conflict of Interests: None.

Funding/Support: None declared by the auhtor.

\section{References}

1. Rubin R. What Happens When COVID-19 Collides With Flu Season? JAMA. 2020;324(10):923-5. doi: 10.1001/jama.2020.15260. [PubMed: 32818229].

2. CDC. Burden of influenza. 2020, [cited 2020 Jul 13]. Available from: https://www.cdc.gov/flu/about/burden/index.html.

3. Adhikari EH, Moreno W, Zofkie AC, MacDonald L, McIntire DD, Collins RRJ, et al. Pregnancy Outcomes Among Women With and Without Severe Acute Respiratory Syndrome Coronavirus 2 Infection. JAMA Netw Open. 2020;3(11). e2029256. doi: 10.1001/jamanetworkopen.2020.29256. [PubMed: 33211113]. [PubMed Central: PMC7677755].

4. Yancy CW. COVID-19 and African Americans. JAMA. 2020;323(19):1891-2. doi: 10.1001/jama.2020.6548. [PubMed: 32293639].

5. Wilson N, Kvalsvig A, Barnard LT, Baker MG. Case-Fatality Risk Estimates for COVID-19 Calculated by Using a Lag Time for Fatality. Emerg Infect Dis.2020;26(6):1339-441. doi:10.3201/eid2606.200320. [PubMed: 32168463]. [PubMed Central: PMC7258483].

6. Cowling BJ, Ali ST, Ng TWY, Tsang TK, Li JCM, Fong MW, et al. Impact assessment of non-pharmaceutical interventions against coronavirus disease 2019 and influenza in Hong Kong: an observational study. Lancet Public Health. 2020;5(5):e279-88. doi: 10.1016/S2468-2667(20)30090-6. [PubMed: 32311320]. [PubMed Central: PMC7164922]. 